\title{
Thermal gasification of agro-industrial residues
}

\author{
P. S. D. Brito, L. F. Rodrigues, L. Calado \& A. S. Oliveira \\ C3i, Interdisciplinary Center for Research and Innovation, \\ Polytechnic Institute of Portalegre, Portugal
}

\begin{abstract}
The farming and agro-industrial sector generates large amounts of organic residues which have a high energetic potential and in most cases are not recovered. Various technologies, including those for energy and agricultural applications, have been considered for the economical valorization of these residues. In this work, we show the results of a study on the potential use of some agro-industrial residues produced in the Portuguese Alto-Alentejo region, in particular, coffee husks, and forest and vineyards residues. The study was conducted in a pilot thermal gasification plant, installed at Portalegre's Industrial Park based on the fluidized bed technology, with a processing capacity of $70 \mathrm{~kg} / \mathrm{h}$, and operating at around $800^{\circ} \mathrm{C}$. The gasification tests were performed continuously for several days, using different waste in order to optimize the heat value and composition of produced syngas. A comparative evaluation of the economic viability of the use of this waste as raw material for the commercial production of synthesis gas and synthetic city gas was made. The results achieved allow the conclusion that the waste biomasses studied show interesting features for use as syngas raw material.
\end{abstract}

Keywords: biofuel, pilot plant, gasification, fluidized bed, syngas.

\section{Introduction}

Bioenergy and biofuels are one of the most important alternatives to fuel production to minimize the continuous depletion of fossil fuel resources and the increasing concerns with environmental issues. Organic living matter, or biomass, in energy terms, is no more than a form of chemical storage of solar electromagnetic energy. When the bonds between carbon, hydrogen and oxygen 
atoms of the organic molecules are broken, during technological processes such as digestion, combustion or gasification, there is a release of the stored chemical energy. It is estimated that the annual global biomass energy storage is approximately $1.33 \times 10^{14} \mathrm{~W}\left(0.26 \mathrm{~W} / \mathrm{m}^{2}\right), 57.14 \%$ of which is produced on earth. It is estimated that the biomass accumulated as energy is of the order of $1.5 \times 10^{22} \mathrm{~J}$. It is obvious that only a part of this biomass can be exploited in technological and economic terms for the production of energy. However, there is a large amount of biomass available that possesses excellent conditions of exploitation, particularly waste from agriculture, forestry and related industries, as well as, from industries and domestic waste in general. According to the European Environmental Agency (EEA), the use of biomass for energy purposes will grow significantly over the coming decades. Predictions point for the end of the century, for a share of about $25 \%$ for bioenergies among all energy sources, namely, oil, natural gas, renewable and nuclear.

Gasification appears to be a promising process to convert biomass in syngas containing methane and hydrogen to be used directly as energy source or as raw material for the production of liquid fuels and other chemicals $[1,2]$. The production of gas with calorific value from materials containing carbon is quite an old technology, that is known since the end of the 19th century. With the development of technologies that use oil as a raw material, the interest in biomass gasification processes declined until the energy crisis of the 70 years of the last century. More recently, due to the discussion on climate change, the Kyoto Protocol commitments and the need to use renewable energy sources, the interest in gasification of biomass is increasing rapidly [3, 4]. Biomass gasification offers advantages over combustion processes since that technology allows the coproduction of minor levels of $\mathrm{NO}_{\mathrm{x}}, \mathrm{SO}_{\mathrm{x}}$, particulates and heavy metals in gaseous emissions and presents a greater potential for reducing $\mathrm{CO}_{2}$ emissions.

Different reactor types have been used to fulfill biomass gasification: fixed bed (updraft, downdraft, or cross-current), fluidized bed, doubled fluidized bed, circulating fluidized bed, entrained flow, etc. $[1,3]$. The use of fluidized bed technology in biomass gasification units makes it possible to use relatively smaller gasifiers and larger capacities than with fixed bed. The higher efficiency of fluidized bed comes from its higher specific reaction area when compared to a fixed bed. Fluidized beds also proved to permit more versatile units in terms of feeding materials of different characteristics and origins, although the relatively high temperatures used to avoid sintering problems of the bed material leads to the formation of tars that complicate operation of equipment due to clogging of equipment and piping [5].

Agroindustry is one of the most important economic activities in the Portugal's Alentejo region, that generates large amounts of residues, in particular, vine prunings, bagasse, coffee husks, forest residues, among others, which require treatment or an adequate recovery to minimize environment impacts and increase the economic value of this waste. A large variety of technologies have been developed over the past decades to deal with this problem. Among the proposed technologies, those oriented towards energy 
recovery, including combustion and gasification of biomasses has attracted much interest [6].

The purpose of this work is to contribute to an assessment of the potential of biomass energy available in the Norte Alentejo region and to study the technical feasibility of the energetic recovery of waste produced in agroindustry through thermal gasification technologies.

\section{Experimental}

The experiments were performed in a gasification pilot plant (Figure 1), which is based on an up-flow fluidized bed gasifier, operated up to $850^{\circ} \mathrm{C}$, under a total pressure below 1 bar and at a maximum pellet feeding rate of $70 \mathrm{~kg} / \mathrm{h}$. In the present work, the results of gasification experiments of different compositions of mixtures of vine pruning and wood pellets are presented.
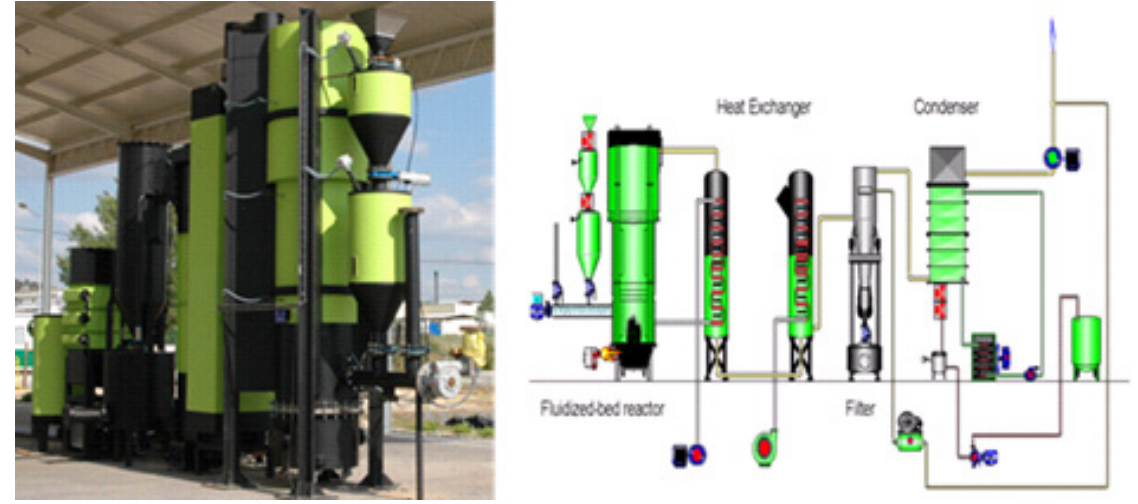

Figure 1: Biomass gasification pilot scale plant at Escola Superior de Tecnologia e Gestão, Instituto Politécnico de Portalegre, Portalegre, Portugal. The main components of the unit are described in the text.

Figure 1 displays a diagram of the biomass gasification pilot scale plant used in the experiments. Main components of the unit are the following: a) Biomass feeding system with two in-line storage tanks that allow the discharge of the biomass into the reactor using an Arquimedes screw at a variable and controllable speed; these two storage tanks act as buffers to avoid the entrance of air through the feeding system; b) Fluidized bed reactor that is a tubular reactor $0.4 \mathrm{~m}$ in diameter and $2.5 \mathrm{~m}$ height, internally coated with ceramic refractory materials; biomass enters the reactor at a height of $0.5 \mathrm{~m}$, from its base, and preheated air enters the reactor coming from the base through a set of diffusers, warranting a flow of about $70 \mathrm{~m}^{3} / \mathrm{h}$; three temperature sensors are installed inside the reactor in order to monitor and control the gasification temperature around $800^{\circ} \mathrm{C}$; syngas leaves the reactor through its top, at about $700^{\circ} \mathrm{C}$; As referred, the reactor operates at negative pressure gradient produced by a vacuum pump installed at the end of the process line; temperature control inside the reactor is a 
determining factor for the gasification process and this control is achieved by tuning the amount of air admitted to the reactor, namely, increasing the air flow rate to increase the reaction temperature; fluidized bed was made of $70 \mathrm{~kg}$ of dolomite. c) Gas cooling system consisting of two heat exchangers; the first exchanger cools the syngas to about $300^{\circ} \mathrm{C}$ using an air co-current flow that enters the unit, and the second heat exchanger cools the syngas down to $150^{\circ} \mathrm{C}$ by a forced flow of air coming from the exterior; d) Cellulosic bag filter that allows the removal of carbon black and ashes particles produced during the gasification process; the cleaning of the filter is made by pressurized syngas injection; black carbon is collected at bottom of the bag filter and is stored in a proper tank; e) Condenser where liquid condensates are removed by cooling the syngas down to room temperature on a third tube heat exchanger.

Tests were made using feeds of different biomasses such as coffee husk, forest residues and vineyards residues, at $800^{\circ} \mathrm{C}$. Feedstock admission rates of 40 and $63 \mathrm{~kg} / \mathrm{h}$ were tested in order to study syngas composition as a function of feedstock composition and operational conditions. Biomasses tested have the elemental composition, in terms of carbon $(\mathrm{C})$, hydrogen $(\mathrm{H})$ and oxygen $(\mathrm{O})$, presented in Table 1. Table 1 presents also some other parameters for the characterization of the biomasses used, namely, humidity, density and Net Heat Values (NHV). The NHV of biomass was determined with an IKA Laboratory Equipment C 200 Calorimeter system.

Table 1: $\quad$ Biomass proprieties.

\begin{tabular}{cccc}
\hline \multicolumn{1}{c}{ Biomass proprieties } & $\begin{array}{c}\text { Forest } \\
\text { residues }\end{array}$ & Coffee husk & $\begin{array}{c}\text { Vines } \\
\text { pruning }\end{array}$ \\
\hline Elementary analysis (\%) & & & \\
$\mathrm{N}$ & 2,4 & 5,2 & 2,6 \\
$\mathrm{C}$ & 43,0 & 40,1 & 41,3 \\
$\mathrm{H}$ & 5,0 & 5,6 & 5,5 \\
$\mathrm{O}$ & 49,6 & 49,1 & 50,6 \\
Humidity $(\%)$ & 11,3 & 25,3 & 13,3 \\
Density $\left(\mathrm{Kg} / \mathrm{m}^{3}\right)$ & 650 & 500 & 265 \\
Net Heat Value $(\mathrm{MJ} / \mathrm{Kg}$ biomass) & 21,2 & 20,9 & 15,1 \\
\hline
\end{tabular}

Syngas analysis was performed in a Varian 450-GC gas chromatograph with two TCD detectors that allow the detection of $\mathrm{H}_{2}, \mathrm{CO}, \mathrm{CO}_{2}, \mathrm{CH}_{4}, \mathrm{O}_{2}, \mathrm{~N}_{2}, \mathrm{C}_{2} \mathrm{H}_{6}$, $\mathrm{C}_{2} \mathrm{H}_{4}$ (equipped respectively with CP81069, CP81071, CP81072, CP81073 and CP81025 Varian GC columns), using helium as carrier gas. Syngas samples for the analysis referred above were collected in appropriate collection and analysis Tedlar ${ }^{\odot}$ bags at the condenser exit every time when the gasification of a given feedstock composition reached its stationary state. Collected syngas samples were injected (within 1 hour after sampling) directly from the sampling bags in the chromatograph using a peristaltic pump operating at is maximum rate and equipped with a Marpren ${ }^{\circledR}$ tube. Chromatographic peaks for the different gases under analysis were identified based on their retention times, and by comparing 
them with the retention times of the same gases in the reference chromatogram of the "custom solution", provided by Varian. Gas mass percentage composition was calculated on the basis of peak areas under chromatographic signal.

\section{Results and discussion}

\subsection{Gasification}

Compositions and net heating value (NHV) of the different syngas produced are presented in Table 2. NHVs were calculated on basis of syngas composition determined by gas chromatography and using the standard combustion heats of the compounds obtained at $25^{\circ} \mathrm{C}$.

Generally, the results show that the produced syngas is relatively rich in carbon monoxide (mass percentage between 8 and 19\%), methane (1 to 5\%) and hydrogen (5 to $13 \%$ ), and therefore these three gases are mainly responsible for the heat content of the syngas produced. NHV's values lie between 2 and 5 $\mathrm{MJ} / \mathrm{kg}$, as can be seen in table 2 . On the other hand, it can be seen that the syngas also contains large amounts of nitrogen (48 to 56\%) and some carbon dioxide (16 to $20 \%$ ) resulting from the partial combustion process that takes place simultaneously with biomass gasification.

The thermal biomass gasification process involves a set of complex chemical reactions that lead to the formation of three fractions: the syngas, ashes (chars) and condensates [10]. The most important fraction, amounting to more than $70 \%$ (w) is made of light gases, namely, $\mathrm{CO}, \mathrm{H}_{2}, \mathrm{CH}_{4}, \mathrm{CO}_{2}$, and $\mathrm{N}_{2}$. In fact it is considered that in the thermal gasification process gases leave the reactor in equilibrium condition. Main equilibrium reactions to be considered are the steam methane reforming reaction (1) and primary water-gas reaction (2):

$$
\begin{aligned}
& \mathrm{CH}_{4}+\mathrm{H}_{2} \mathrm{O} \leftrightarrow 3 \mathrm{H}_{2}+\mathrm{CO} \\
& \mathrm{CO}+\mathrm{H}_{2} \mathrm{O} \leftrightarrow \mathrm{H}_{2}+\mathrm{CO}_{2}
\end{aligned}
$$

In a first approach, it may be said that the gas composition, the syngas fraction and the amount of ashes and condensates are a function of biomass nature and its elemental composition, and of the gasification operational conditions, in particular, of the process temperature.

Generically, the results obtained with the various biomass studied shows a relation between the biomass calorific value and the calorific value of the syngas obtained: higher biomass calorific value results in higher calorific syngas production. In fact, it turns out that the syngas formed with the highest calorific value was obtained by gasification offorest residues, a more energetic material. This relationship between the biomass calorific content and the syngas NHV can be explained considering first, that the biomass calorific value is related to the amount of carbon and hydrogen present in the biomass, i.e. an increased amount of carbon and hydrogen, lead to higher calorific value, and second, a larger amount of these two elements allows production of larger quantities of hydrogen and carbon monoxide, the major contributors for the calorific value of the syngas. 


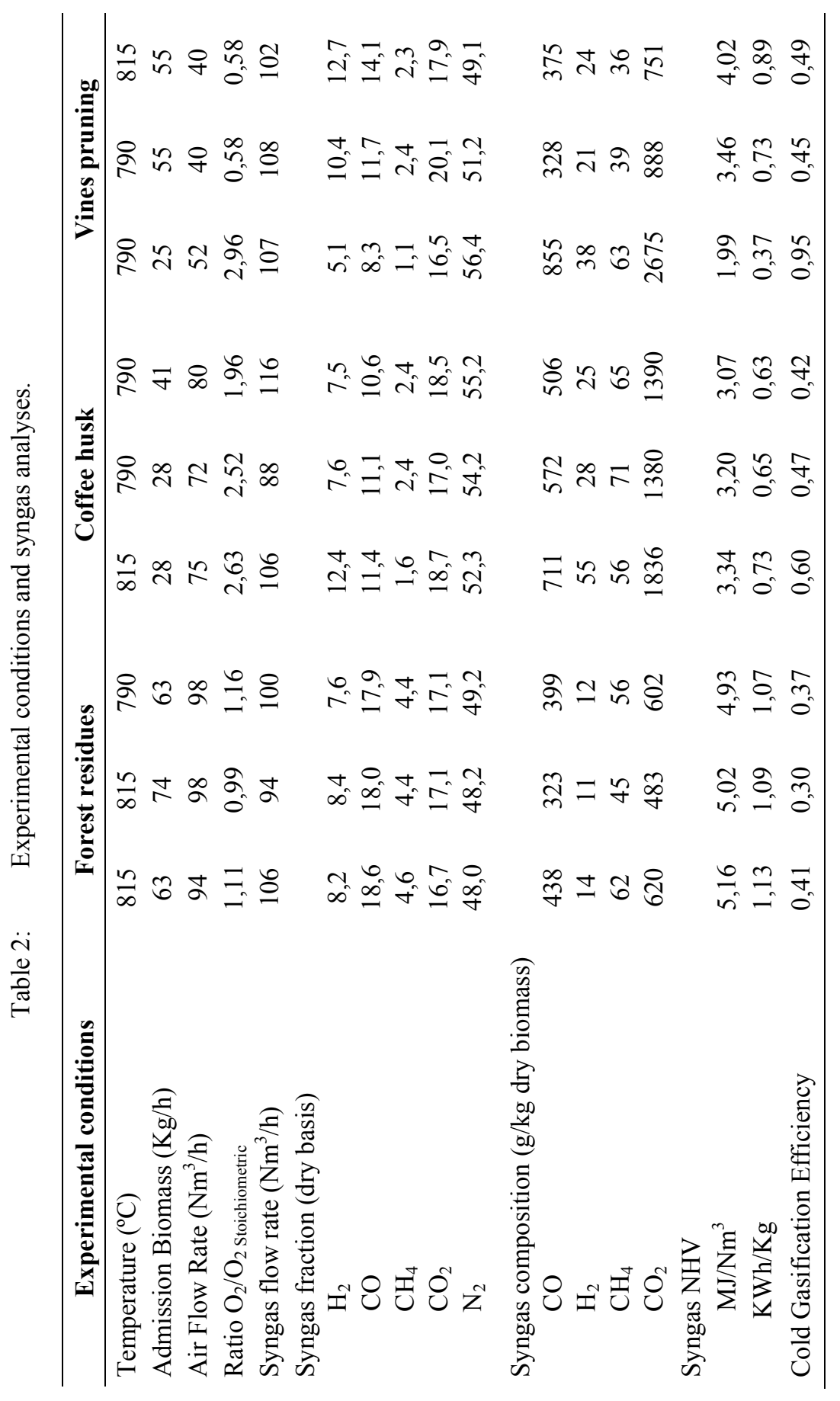


Analysis of the dependence of producer gas properties on conditions of biomass admission shows that for all biomasses studied there is a decrease in syngas calorific value with increasing quantity of biomass admitted. This is consistent with the fact that a faster acceptance of biomass by the reactor reduces the extent of reaction and equilibria represented by equations (1) and (2) are not attained.

For forest residues and coffee husks, an increase in gasification temperature promoted the formation of a syngas with higher hydrogen and carbon monoxide contents and, consequently higher NHV of the syngas. This could be a result of a shifting in the gasification equilibrium, since the reaction represented by equation (2) is endoenergetic and, therefore favoured by a temperature increase. For these residues cold gasification efficiency values are greater than 0.3 and increase with increasing gasification temperature and decreasing oxygen to biomass ratio.

Results of the study of vine pruning gasification (table 2) shows a decrease in the NHV when the gasification temperature is increased. It should be noted that the decreases in NHV were accompanied by decreases in carbon monoxide production and a consequent increase in carbon dioxide content in the producer syngas.

\subsection{Economic evaluation}

In this section the achieved results are analyzed in an economic perspective in a framework of a demand for alternative energy sources to fossil fuels in order to minimize environmental problems and make the full use of the potential of this technology. The analysis will focus on Portuguese Alto-Alentejo main industries, in particular, agro-industries, which are in essence small companies with a high territorial dispersion. In this approach the search for alternative energy sources must imply a strategy to develop local energy sources, in particular, from industrial waste, in order to obtain a decentralized generation of electricity, liquid or gaseous fuels. For analysis purposes it will be considered that the aim would be the use of thermal gasification to produce a gaseous fuel to substitute natural gas utilized by many regional industries.

The analysis was based on the results obtained in this work on gasification of different biomasses, in particular, on the composition and calorific value of syngas obtained and on present prices of natural gas at the market place. It was assumed a daily production of 1 ton of waste. Table 3 shows the parameters used in the calculation and the results in terms of annual return for each of the biomasses.

Assuming that a gasification plant for a capacity of 1 ton per day has an investment cost of around $80 \mathrm{k} €$, constant prices, and that the initial investment would be amortized over about 5-6 years, the average value for other renewable energies systems, such as photovoltaic and micro-wind energy. 
Table 3: $\quad$ Economic evaluations for biomass gasification unit.

\begin{tabular}{lccc}
\hline \multicolumn{1}{c}{ Parameters } & & & \\
\hline Biomass (ton/day) & & 1 & \\
Price $(€ / \mathrm{KWh})$ & & 0,0501 & \\
Fixed costs $(€ /$ month) & & 10 & \\
Energy outflow in operation (\%) & & 15 & \\
\hline \multicolumn{1}{c}{ Biomass } & Forest & $\begin{array}{c}\text { Coffee } \\
\text { husk }\end{array}$ & $\begin{array}{c}\text { Vines } \\
\text { pruning }\end{array}$ \\
\hline Advantage $(\mathrm{k} € /$ year) & 17,6 & 17,1 & 16,7 \\
\hline
\end{tabular}

\section{Conclusions}

\section{Based on the results achieved in this work it may be concluded that:}

- The increase of gasification temperature and feedstock admission rate improved the syngas Net Heat Value (NHV);

- the economic evaluation for the recovery of agroindustrial waste by thermal gasification compared with the present costs for natural gas in the AltoAlentejo region industries shows clear economic potentialities for the application of the biomass gasification technology considered.

\section{Acknowledgements}

The authors thank the U.E. Commission, POCTEC Program, ALTERCEXA Project, for financial support.

\section{References}

[1] Peter McKendry, "Energy production from biomass (part 3): gasification technologies", Bioresource Technology 83 (2002) 55

[2] A. K. Rajvanshi, "Biomass Gasification" in "Alternative Energy in Agriculture", Vol. II, Ed. D.Y. Goswami, CRC Press, 1986, 83

[3] M. Balat, "Gasification of Biomass to Produce Gaseous Products", Energy Sources, A 31 (2009) 516

[4] Peter McKendry, "Energy production from biomass (part 3): gasification technologies", Bioresource Technology 83 (2002) 55

[5] M.J. Prins, "Thermodynamic analysis of biomass gasification and torrefaction", $\mathrm{PhD}$ Thesis at Technische Universiteit Eindhoven, Proefschrift, 2005

[6] L. Fiori, L. Florio, "Gasification and Combustion of Grape Marc: Comparison Among Different Scenarios”, Waste Biomass Valor 1 (2010) 191 